\title{
Sistema de Informacion que Utiliza Datos del Proceso de Fabricacion de un Controlador Logico Programable
}

\author{
Alicia G. Valdez Menchaca, PhD \\ Juan Carrillo Gándara, M. I. \\ Laura Vazquez \\ Griselda Cortes \\ Sergio Castaneda \\ Adriana Martinez \\ Universidad Autónoma de Coahuila, México \\ Jose Luis Cendejas Valdez \\ Universidad Tecnologica de Morelia, Mexico
}

Doi:10.19044/esj.2021.v17n17p98

Submitted: 30 January 2021

Accepted: 07 April 2021

Published: 31 May 2021
Copyright 2021 Author(s)

Under Creative Commons BY-NC-ND

4.0 OPEN ACCESS

Cite As:

Valdez Menchaca A.G., Gándara J.C., Vazquez L., Cortes G., Castaneda S., Martinez A. \& Cendejas Valdez J.L. (2021). Sistema de Informacion que Utiliza Datos del Proceso de Fabricacion de un Controlador Logico Programable. European Scientific Journal, ESJ, 17(17), 98. https://doi.org/10.19044/esj.2021.v17n17p98

\section{Resume}

En la actualidad, las empresas requieren de la obtención de datos precisos, análisis que apoye en la toma de decisiones y en la mejora continua; estos procesos han llegado a formar parte de la visión de negocios de la gerencia empresarial sobre la cual están orientados los objetivos de negocios para poder ser y permanecer competitivos. Las empresas de manufactura tienen la necesidad de obtener y analizar datos relacionados a los procesos de producción para determinar el nivel de eficiencia de estos y en base a la información obtenida tomar decisiones que ayuden a la empresa a mejorar o corregir situaciones que puedan ser de carácter humano, operativo, procedural o de equipo. En este proyecto de desarrollo de software se ha diseñado e implementado un sistema de información, con el método de cascada, para una empresa de manufactura localizada en la frontera norte de México con Estados Unidos en el sector del cuidado de la salud; el sistema utiliza datos desde un controlador lógico programable, en el cual se obtienen datos para su monitoreo 
como: alarmas de la máquina, conteo de eventos, duración de cada alarma y velocidad de producción. Dando como resultado información de los códigos de producción, turno, día y hora; además de medir de forma precisa la eficiencia de la máquina, así como la detección de tendencias y la creación de alertas oportunas hacia personas técnicas para la solución de problemas. El sistema consta de un análisis de velocidad y carga de trabajo del procesador del PLC, desarrollo de la conexión entre el procesador y el sistema, preparación del sistema para el traspaso de información, programación de la base de datos en Access y el diseño de la interfaz gráfica en Visual Basic. Net. Entre los logros más importantes obtenidos de la implementación del sistema de información se encuentran: Disponibilidad de la información en tiempo real y obtención de datos históricos de las variables de producción del equipo, mejoramiento de las tareas de supervisión y control de procesos, así como el envío de alertas a usuarios específicos para reacción adecuada a eventos de fallas.

Palabras claves : Sistemas de información, PLC, Análisis de datos

\title{
Information System Using Data from the Manufacturing Process of a Programmable Logic Controller
}

\author{
Alicia G. Valdez Menchaca, PhD \\ Juan Carrillo Gándara, M. I. \\ Laura Vazquez \\ Griselda Cortes \\ Sergio Castaneda \\ Adriana Martinez. \\ Universidad Autónoma de Coahuila, México \\ Jose Luis Cendejas Valdez. \\ Universidad Tecnologica de Morelia, Mexico
}

\begin{abstract}
Currently, companies require obtaining accurate data, analysis that supports decision-making and continuous improvement; these processes have become part of the business management vision on which business objectives are oriented to be and remain competitive. Manufacturing companies have the need to obtain and analyze data related to production processes to determine their level of efficiency and based on the information obtained, make decisions that help the company improve or correct situations that may be of human, operational, procedural or equipment. In this software development project, with the cascade method, an information system has been designed and
\end{abstract}


implemented, for a manufacturing company located on the northern border of Mexico with the United States in the health care sector; the system uses data from a programmable logic controller, in which data such as: machine alarms, event count, duration of each alarm, and production speed were obtained for monitoring. Resulting in information on the production codes, shift, day and hour; in addition to accurately measuring the efficiency of the machine, as well as the detection of trends and the creation of timely alerts to technical personnel for troubleshooting. The system consists of an analysis of the speed and workload of the PLC processor, development of the connection between the processor and the system, preparation of the system for the transfer of information, programming of the database and the design of a graphical interface in Visual Basic.Net . Among the most important achievements obtained from the implementation of the information system are: Availability of information in real time and obtaining historical data from production variables, improvement of supervision tasks and process control, as well such as sending alerts to specific users for adequate reaction to failure events.

Keywords: Information systems, PLC, Data analysis

\section{Introduction}

El conocimiento es el recurso primario de los individuos y la economía en general. Toda organización, para garantizar su estabilidad, eficiencia y eficacia, debe incorporar tres prácticas sistemáticas, la mejora continua en actividades y procesos, aprender a explotar todos sus conocimientos y aprender a innovar. Un elemento común de estas prácticas sistemáticas lo constituye la información (Laudon \& Laudon, 2012).

Diversos autores han desarrollado sistemas industriales para la mejora de procesos (Caicedo et al., 2017), construcción de equipos complejos (Orna et al., 2017), entre otros.

La importancia del aprovechamiento de los recursos económicos en las empresas (Paredes et al., 2019), hace que los sistemas de información apoyen en la optimización de los recursos y la disminución de riesgos ocupacionales (Carrera et al., 2019). Mientras que la inclusión de las tecnologías de información en los procesos productivos de las empresas y las instituciones es cada vez más alta (Tobar, Frías, Romero, \& Barrientos, 2019), (Yasaca et al., 2019).

Los sistemas de información han revolucionado el entorno empresarial, organizacional e institucional. Ahora prácticamente es imposible competir, ser eficiente y tener éxito, sin tener información eficaz en el momento adecuado (Amaya, 2018). Estos sistemas deben estar compuestos por: personas, hardware, software, datos y procedimientos (McLeod \& Schell, 2001). Los sistemas de información en línea son sistemas automatizados que 
analizan en tiempo real los datos obtenidos por los procesos para apoyar a los usuarios a adoptar decisiones de negocios, incorporando nuevas funcionalidades para el análisis de datos (Silberschatz, Korth, \& Sudarshan, 2002). De lo anterior se deduce, que las organizaciones que deseen introducir un nuevo sistema de información deben realizar un diagnóstico muy cuidadoso y detallado de los usuarios y de sus necesidades, previo a su implementación; para producir un sistema adecuado que promueva efectos positivos y exitosos. Esto les permitirá a las organizaciones tener la suficiente flexibilidad para proveer bienes y servicios de calidad, acorde con las exigencias de su entorno (Pressman, 2010), (Ozz, 2017).

La empresa donde se desarrolló el proyecto se encuentra localizada en la frontera norte de México con los Estados Unidos, forma parte del sector del cuidado de la salud; teniendo como objetivo la manufactura de productos médicos innovadores utilizados en hospitales, centros de salud e instalaciones industriales en todo el mundo.

Los productos elaborados son de un solo uso, el $100 \%$ de la producción es exportada hacia Estados Unidos, donde se distribuye a los principales puntos de venta o consumo donde figuran Estados Unidos, Asia y Europa.

La necesidad del sistema de información, obtención y análisis de datos que permita obtener las variables de fabricación para la disponibilidad de la información en tiempo real, así como el mejoramiento de la supervisión y control de procesos que requiere la empresa está enfocada principalmente al proceso de fabricación de sábanas quirúrgicas, el cual es un proceso complejo debido a la gran cantidad de componentes eléctricos, neumáticos y lo extenso del programa del Controlador Lógico Programable (PLC); este se basa en el desarrollo de equipos capaces de procesar y memorizar variables físicas, que constituyen sistemas de tratamiento de la información; estos sistemas son capaces de recibir información de productos o procesos industriales (Mandado, Acevedo, Fernández, \& Armesto, 2009). Por lo cual la obtención de datos del PLC es la principal solución en este tipo de sistemas de información.

En muchas aplicaciones de automatización industrial, utilizan el control de procesos para manejar una aplicación que involucra PLC, sistemas de control distribuido, controles de supervisión, sistemas de adquisición de datos (SCADA), entre otros, para lograr controlar un sistema específico en tiempo real (Bagal, Kadu, Vikhe, \& Parvat, 2018).

Este proceso de fabricación es llevado a cabo por una maquina llamada "Fan Fold Drape Machine" (Rowley, 2021), el propósito de esta máquina es el de convertir un rollo de material de fibras plásticas en paquetes de sábanas identificadas por código y tamaño, plenamente dobladas con un doblez tipo abanico de allí su nombre. 
Esta máquina está controlada por un PLC de la marca Allen-Bradley modelo SLC-500 con un procesador 5/04 (RockwellAutomation, 2021), el cual se comunica con cinco servo controladores modelo 1394 de cuatro ejes cada uno, ya que se requiere de precisión en los movimientos de la máquina para así colocar la sábana en las posiciones requeridas para sus dobleces, los cuales son efectuados por cilindros neumáticos. El desempeño de cada uno de los cilindros neumáticos y posicionamiento de la sábana juegan un papel clave en la producción de esta máquina.

Se compone de una interfaz tipo Panelview 1000, la cual se utiliza para accionar algunas opciones y funciones de la máquina, selección de códigos de producción, cambio y selección de velocidades de producción, accionamiento manual de cilindros y movimientos de la máquina; así como mostrar reportes en pantalla como razones por la cual la máquina detiene la producción, conteo de tiempo en minutos del tiempo caído por cada falla y número de veces que cada falla se presenta en el turno.

\section{Revisión de la literatura}

Un sistema de información es un conjunto de elementos que interactúan entre sí con el fin de apoyar las actividades de una empresa o un negocio. Estos elementos son de una naturaleza diversa y normalmente incluyen (Hoffer, George, \& Valacich, 2011).

- El equipo computacional, es decir, el hardware necesario para que el sistema de información pueda operar. Lo constituyen las computadoras y el equipo periférico que puede conectarse a ellas.

- El recurso humano que interactúa con el sistema de información, el cual está formado por las personas que utilizan el sistema, alimentándolo con datos o utilizando los resultados que genere.

- Los datos o información fuente son todas las entradas que necesita el sistema de información para generar como resultado la información que se desea.

- Los programas que son procesados y producen diferentes tipos de resultados. Los programas son la parte del software del sistema de información que hará que los datos de entrada introducidos sean procesados correctamente y generen los resultados que se esperan.

Un sistema de información realiza cuatro actividades básicas: entrada, almacenamiento, procesamiento y salida de información. En la Fig. 1 se muestra el diseño conceptual del sistema de información. 


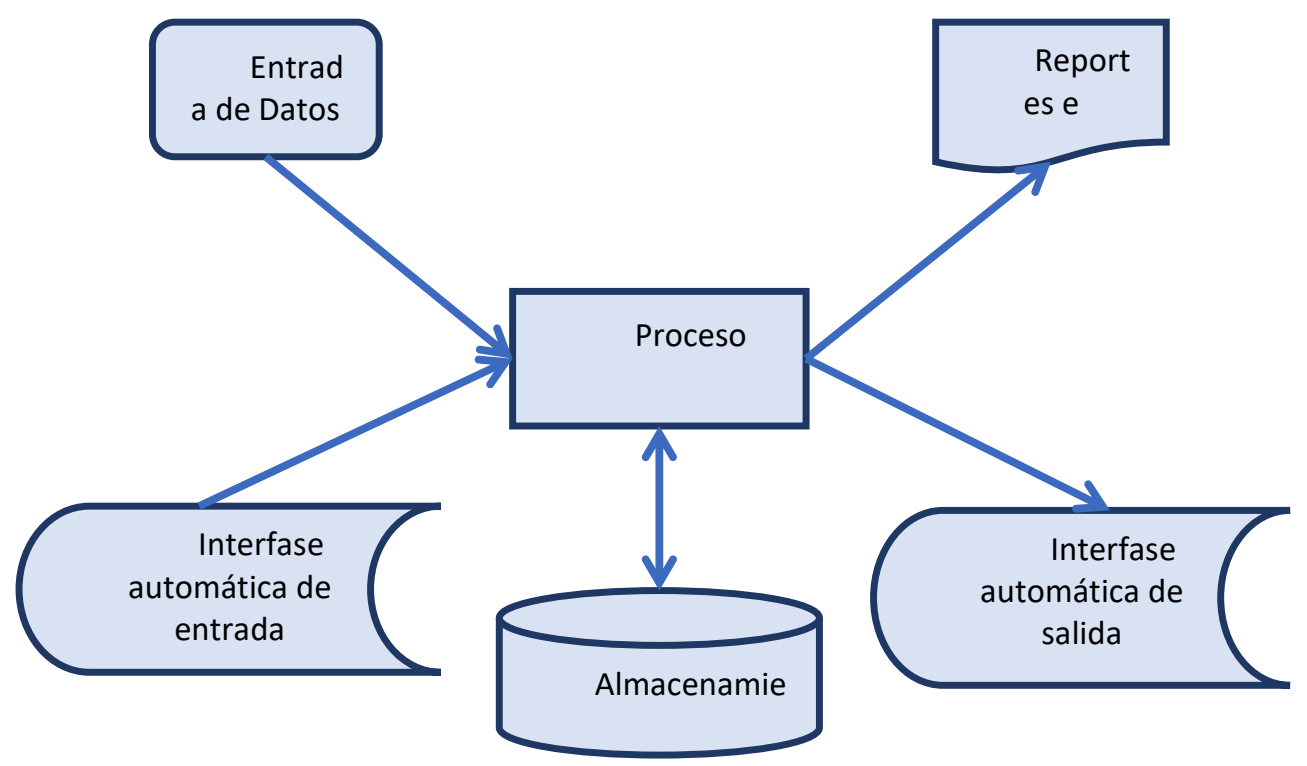

Figura 1. Diseño conceptual del sistema de información, fuente: elaboración propia

Los PLC iniciaron como una solución al control de circuitos complejos de automatización, se puede argumentar que es un aparato electrónico que sustituye los circuitos auxiliares o de mando de los sistemas automáticos, en el PLC se conectan los dispositivos de señales (pulsadores) y los actuadores (bobinas de contactores, lámparas y pequeños receptores, entre otros) (Dana \& Yendol-Hoppey, 2016).

Las computadoras personales están comenzando a reemplazar al PLC en algunos casos, también se cuentan con SLC o sistemas lógicos programables, que representan la siguiente generación de los PLC, los SLC cuentan con muchas capacidades que los hacen un sistema más robusto al utilizar comunicación ethernet industrial, comunicación e integración con sistemas de control de movimiento, drives de AC, manejo de sistemas de seguridad, entre otros (Tocci \& Widmer, 2003).

La estructura externa de un PLC industrial se refiere al aspecto físico exterior o los elementos que lo integran, como se muestra en la Fig. 2. 


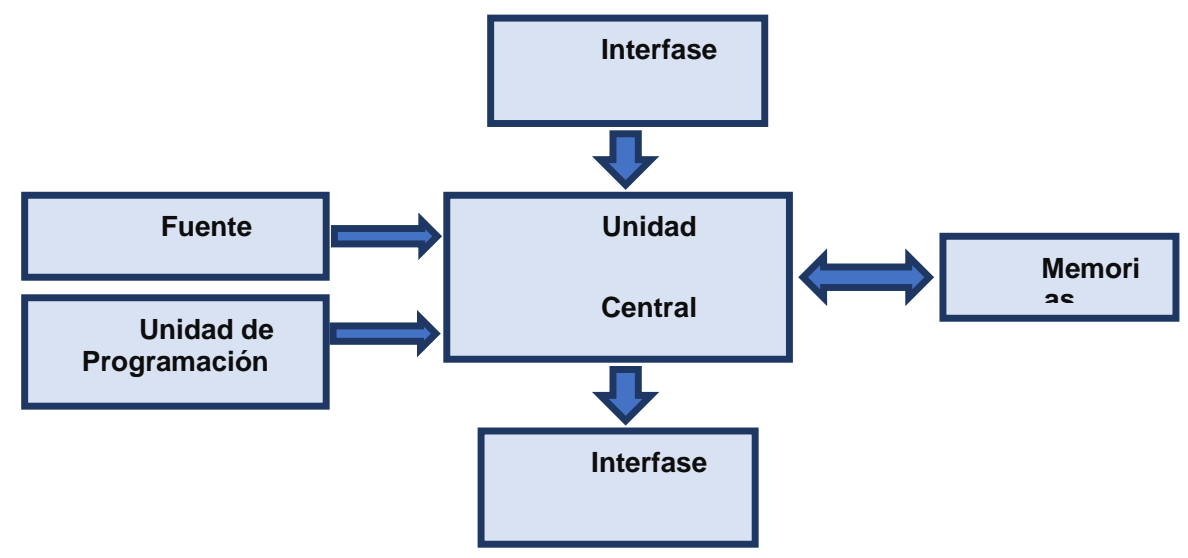

Figura 2. Estructura de un PLC, fuente: elaboración propia

Los bloques de un PLC son (Hermosillo, 2019):

\section{Unidad de Programación}

Es el conjunto de medios, hardware y software mediante los cuales el programador introduce y depura sobre las secuencias de instrucciones (en uno u otro lenguaje) que constituyen el programa a ejecutar.

\section{Fuente de Alimentación}

La fuente de alimentación proporciona los voltajes necesarios para el funcionamiento de los distintos circuitos del sistema. La alimentación al CPU puede ser de $24 \mathrm{Vcd}$ o de 110 / $220 \mathrm{Vca}$. En cualquier caso, es el propio CPU el que alimenta las interfaces conectadas a través del bus interno. La fuente de alimentación del PLC puede incorporar una batería de reserva, que se utiliza para el mantenimiento de algunas posiciones internas y del programa usuario en memoria RAM, o cuando falla la alimentación o se apaga el PLC.

\section{Unidad Central de Procesamiento}

El CPU (Central Procesing Unit) es la parte inteligente del sistema. Interpreta las instrucciones del programa de usuario y consulta el estado de las entradas. Dependiendo de dichos estados y del programa, ordena la activación de las salidas deseadas. Está constituida por los siguientes elementos:

- Procesador

- Memoria monitor del sistema

- Circuitos auxiliares 


\section{Procesador}

Está constituido por el microprocesador, el reloj (generador de onda cuadrada) y algún chip auxiliar. El microprocesador es un circuito integrado (chip), que realiza una gran cantidad de operaciones, que podemos agrupar en:

- Operaciones de tipo lógico

- Operaciones de tipo aritmético.

- Operación de lectura y modificación de datos.

- Operaciones de entrada - salida

- Operaciones de control de la transferencia de la información dentro del PLC.

Para que el microprocesador pueda realizar todas estas operaciones está dotado de unos circuitos internos que son los siguientes:

- Circuitos de la unidad aritmética y lógica o ALU: Es la parte donde se realizan los cálculos y las decisiones lógicas para controlar el PLC.

- Circuitos de la unidad de control (UC) o decodificador de instrucciones: Decodifica las instrucciones leídas en memoria y se generan las señales de control.

- Acumulador: Es la encargada de almacenar el resultado de la última operación realizada por el ALU.

- Flags: Indicadores de resultado, que pueden ser consultados por el programa.

- Contador de programa: Encargado de la lectura de las instrucciones del usuario.

- Bus (interno): No son circuitos en sí, sino zonas conductoras en paralelo que transmiten datos, direcciones, instrucciones y señales de control entre las diferentes partes del PLC

\section{Memoria monitor del sistema}

Es una memoria de tipo ROM, Lectura y escritura en las interfaces de E/S. contiene las siguientes rutinas, incluidas por el fabricante.

- Inicialización tras conectarse a voltaje o reset.

- Rutinas de pruebas y de respuesta a error de funcionamiento.

- Intercambio de información con unidades exteriores.

- Lectura y escritura en las interfaces de E/S.

- Funciones básicas de la CPU.

En la memoria ROM del sistema, el fabricante graba una serie de programas ejecutivos, software del sistema y es a estos programas a los que accederá el procesador para realizar las funciones. 
El software del sistema del PLC consta de una serie de funciones básicas que realiza en determinados tiempos de cada ciclo. En general cada PLC contiene y realiza las siguientes funciones:

- Vigilar que el tiempo de ejecución del programa de usuario, no exceda de un determinado tiempo máximo. A esta función se le denomina Watchdog.

- Ejecutar el Programa del usuario.

- Crear una imagen de las entradas, ya que el programa de usuario no debe acceder directamente a dichas entradas.

- Renovar el estado de las salidas en función de la imagen de estas, obtenida al final del ciclo de ejecución del programa usuario.

- Chequeo del sistema.

\section{Interfase de salida}

En el control de un proceso automatizado, es imprescindible un dialogo entre operador-máquina junto con una comunicación entre la máquina y el PLC, estas comunicaciones se establecerán por medio del conjunto de entradas y salidas.

Todas las señales provenientes del campo son informadas a la CPU, luego de ser tomadas por los captores de entradas, y a su vez, las órdenes generadas por el CPU son comunicadas a los elementos del proceso bajo control por medio de las interfaces de salida.

Las señales digitales o discretas como los interruptores son simplemente una señal de 1 ó 0, Verdadero o Falso, respectivamente. Los interruptores son ejemplos de dispositivos que proporcionan una señal discreta, que son enviadas usando el voltaje, donde un rango específico corresponderá al Verdadero y otro rango al False. Un PLC puede utilizar $24 \mathrm{Vcd}$ en la $\mathrm{E} / \mathrm{S}$ donde valores superiores a $22 \mathrm{~V}$ representan un 1 , y valores inferiores a $2 \mathrm{~V}$ representan un 0 .

Inicialmente los PLC solo tenían E/S discretas. A medida que la complejidad de los PLC aumenta, es necesario contar con otro tipo de interfaces que puedan interpretar señales analógicas provenientes del proceso y emitirlas como salidas. Las señales analógicas son como controles de volúmenes, con un rango de valores entre 0 y el tope de escala. Esto es normalmente interpretado con valores enteros por el PLC, con varios rangos de precisión dependiendo del dispositivo o del número de bits disponibles para almacenar los datos.

Presión, temperatura, flujo, y peso son normalmente representados por señales analógicas. Las señales analógicas pueden usar voltaje o corriente con una magnitud proporcional al valor de la señal que procesamos. A medida que los requerimientos de control se hacen más complicados, aparecen los sistemas inteligentes, periféricos cuentan con un microprocesador propio, que 
descargan en parte el trabajo del CPU, para hacer más rápida la ejecución del programa del usuario.

La Fig. 3 muestra el diseño general del sistema de información propuesto para la máquina dobladora de sábanas quirúrgicas.

El software que se utilizó para el desarrollo es Visual Basic.Net ya que se cuenta con licencias en la empresa por lo que existe una disponibilidad en la empresa, es de fácil acceso, actualizado y compatible con otros sistemas de información de la compañía.

\section{Selección del equipo y software de adquisición de datos}

Uno de los primeros pasos necesarios para poder realizar una adquisición de datos exitosa, consiste en la búsqueda, así como aplicación de dispositivos y protocolos de comunicación PLC- PC-Servidor confiables. Para la selección del equipo para la lectura de datos del PLC, se consideraron las diferentes opciones compatibles con los SLC-500. Entre las opciones de protocolos de comunicación se tienen: Ethernet industrial, DH+, DH-485 y RS-232, como se muestra en la Tabla 1.

En los niveles industriales las máquinas individuales actúan como una completa celda de trabajo, cada máquina tiene sus señales propias ya sean sensores analógicos y discretos para monitorear variables del proceso, como por ejemplo temperatura. Los valores de estos sensores son el punto de partida para los lazos de control orquestado por los PLC's.

Los PLC's proveen señales de control a actuadores, para activar y desactivar calentadores, válvulas entre otros. A este tipo de funcionamiento se le conoce como nivel de campo o "Fieldbus". 


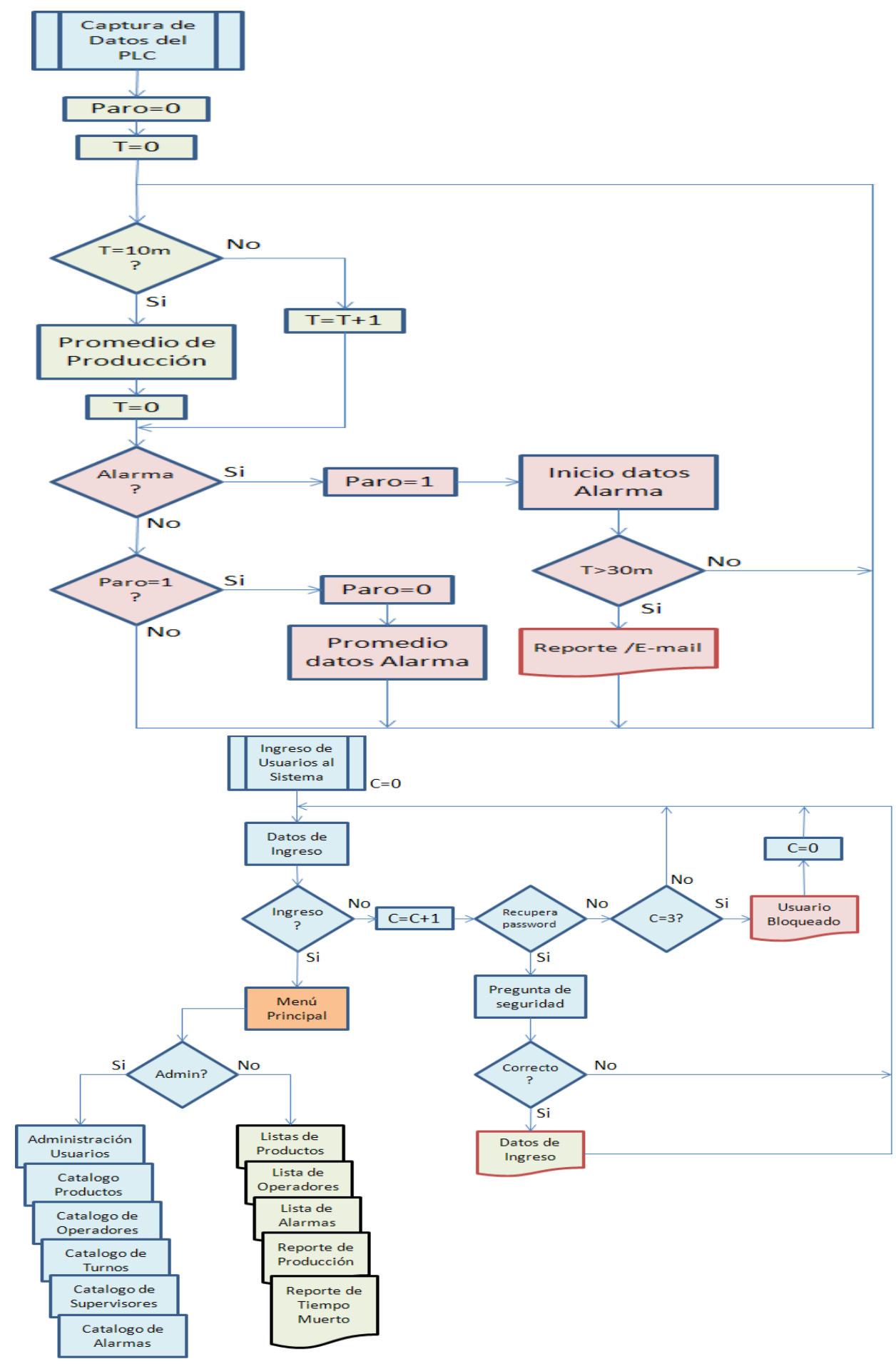

Figura 3. Diseño general del sistema de información para máquina dobladora de sábanas 
Ethernet: Es una red local que provee comunicación con otros dispositivos a una velocidad de $10 \mathrm{Mbps}$, es la comunicación más rápida y confiable para el SLC-500.

DH+ (Data Highway Plus): Es una red local que provee comunicación a $230.4 \mathrm{Kbps}$ se usa a través del canal 1 de un procesador y requiere de una tarjeta 1784-PCMK, -KT, -KTX o -KTXD en la PC para la comunicación, no es apta para comunicación a largas distancias, ya que es muy susceptible a ruidos eléctricos, se requieren consideraciones especiales para la instalación en equipo eléctrico.

DH-485: Es un grupo de dispositivos conectados a un cable de comunicación permitiendo el intercambio de información, se usa a través del canal 1, requiere un 1747-PIC, 1784-PCMK, -KTX o-KTXD y se comunica a 19,200bps.

RS-232: Permite comunicarse con la PC, usa el canal 0 y se comunica a 19,200 bps.

\begin{tabular}{|l|l|l|l|l|l|}
\hline $\begin{array}{l}\text { Opciones de } \\
\text { Comunicaciones }\end{array}$ & $\mathbf{5 / 0 1}$ & $\mathbf{5 / 0 2}$ & $\mathbf{5 / 0 3}$ & $\mathbf{5 / 0 4}$ & $\mathbf{5 / 0 5}$ \\
\hline Ethernet & & & & & $\checkmark$ \\
\hline DH+ & & & & $\checkmark$ & \\
\hline DH-485 & $\checkmark$ & $\checkmark$ & $\checkmark$ & $\checkmark$ & $\checkmark$ \\
\hline RS-232 & & & $\checkmark$ & $\checkmark$ & $\checkmark$ \\
\hline
\end{tabular}

Tabla 1. Opciones de protocolos de comunicación disponible para procesador del tipo SLC500

Por lo tanto, se ha considerado la utilización de la comunicación ethernet como protocolo de comunicación a utilizar sería la más apta para un correcto funcionamiento del sistema. Sin embargo, el procesador 5/04 no es compatible con comunicación ethernet, por lo que se requirió un módulo especial para poder lograr esta comunicación. por lo que se utiliza un módulo especial para este propósito. El módulo debe ser insertado en una ranura disponible del rack, en este caso en particular el módulo se inserta en la ranura o slot 5, como se muestra en la Fig. 4. 

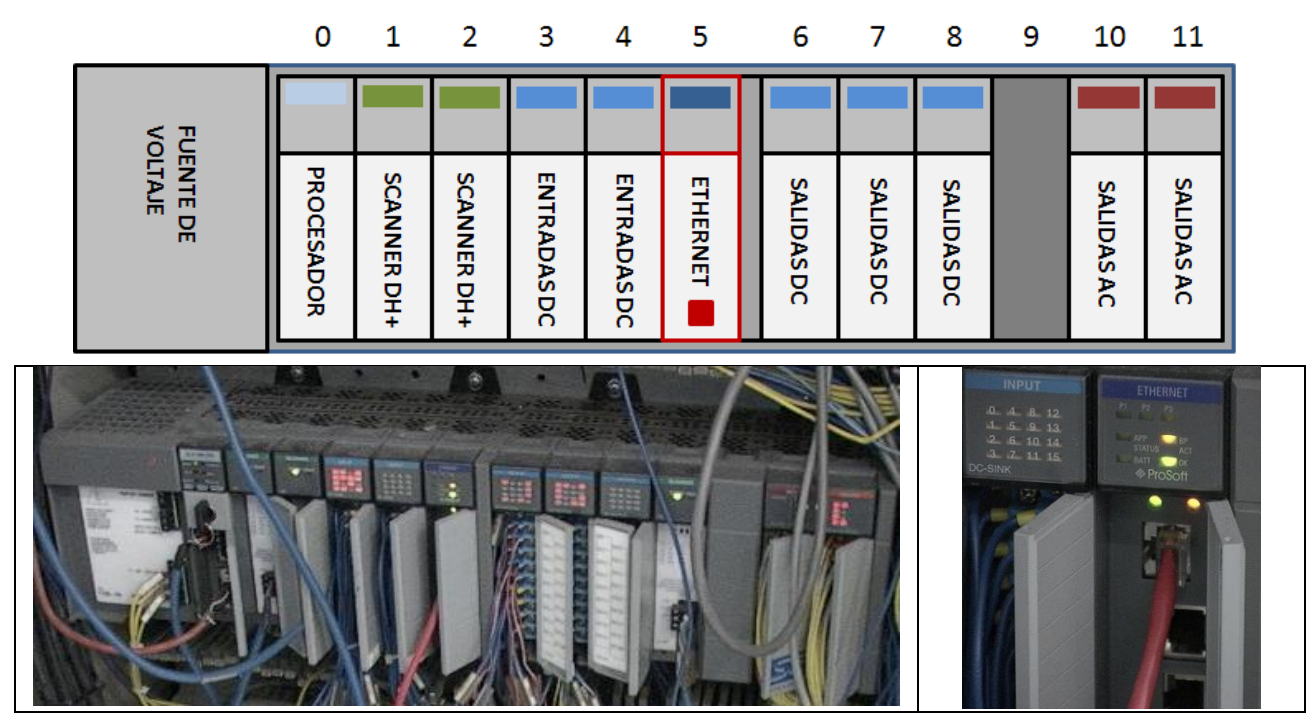

Figura 4. Imagen del SLC-500 de la máquina dobladora de sabanas

El módulo de ethernet se utiliza para vincular los PLC's individuales con un sistema de recolección de datos y control. La función primaria de este sistema es el de recolectar datos del proceso para revisiones de administración y estadísticas, proveer control de la información para mantener todas las celdas individuales coordinadas y monitorear la operación entera en busca de anomalías que requieran la intervención humana.

Los sistemas de recolección de datos SCADA (Supervisory Control And Data Adquisition) son aplicaciones de software, diseñadas con la finalidad de controlar y supervisar procesos a distancia (Zhang, Wang, Xiang, \& Ten, 2015), (Abdulsattar, Mustafa, \& Hadi, 2019). Se basan en la adquisición de datos de los procesos remotos por medio de una aplicación de software, especialmente diseñada para funcionar sobre computadoras en el control de la producción. Cada uno de los módulos SCADA involucran muchos subsistemas, por ejemplo, la adquisición de los datos puede estar a cargo de un PLC, el cual toma las señales y las envía a las estaciones remotas usando un protocolo determinado, otra forma podría ser que una computadora realice la adquisición vía un hardware especializado y luego esa información la transmita hacia un equipo de radio vía su puerto serial.

Un software SCADA debe ser capaz de ofrecer al sistema (Thomas \& McDonalds, 2015):

- Posibilidad de crear paneles de alarma, que exigen la presencia del operador para reconocer una parada o situación de alarma, con registro de incidencias.

- Generación de datos históricos de las señales de planta, que pueden ser transferidos para su proceso sobre una hoja de cálculo. 
- Ejecución de programas, que modifican la ley de control, o incluso anular o modificar las tareas asociadas al autómata, bajo ciertas condiciones.

- Posibilidad de programación numérica, que permite realizar cálculos aritméticos de elevada resolución sobre la CPU del ordenador.

\section{Metodología}

La metodología utilizada para el diseño, desarrollo e implementación del sistema de información para el monitoreo de datos obtenidos de un PLC de una máquina dobladora de sábanas, donde se ha incluido un conjunto de actividades entre las cuáles se encuentran: Análisis y diseño general del sistema de información, selección del software para desarrollo y modelación de la base de datos, desarrollo del algoritmo general del sistema, diagramas generales de flujo, así como los requerimientos del hardware y software.

Se ha utilizado el método de cascada(Pressman, 2010) para el ciclo completo de desarrollo.

Las mejoras que se han logrado de este desarrollo de software han sido la utilización de datos en tiempo real de la máquina, se han podido realizar promedios y consultas de los datos sin comprometer la conexión hacia el PLC; visualizando los datos de producción y tiempos muertos relacionando algunas variables como operadores, turnos y productos; buscando patrones de comportamiento y tendencias de los datos.

\section{Diseño del sistema de información}

El sistema de información se ha diseñado por módulos, donde el módulo que representa al SLC-500 es la forma en que los datos o estados de las variables del PLC son transferidas a registros de memoria, los cuales son leídos directamente a través de RSLinx. En el siguiente cuadro que corresponde al Visual Basic.Net contiene la lectura de variables del PLC como por ejemplos datos de producción: como velocidad de la máquina, numero de producto, tiempo activo, así como un código de alarma; todo esto se realiza creando una conexión a RSLinx.

Los valores de las variables son entonces transferidos a variables internas dentro del código del programa .Net, de igual forma establece una conexión a la base de datos en Access para leer datos de catálogos como turno, descripciones de alarma, producto y operador, para tener en una transacción toda la información que pueda proporcionar valor de negocio a la empresa.

Después todos los datos son almacenados en la base de datos para tener datos históricos, generación de reportes de producción, tiempo muerto, buscar tendencias y ayudar a la empresa en la toma de decisiones, que es la intención principal del sistema. 
El sistema de información está basado en dos archivos principales, un ejecutable compilado del código .Net, el cual su función es realizar las conexiones hacia el PLC y la base de datos para recolectar la información requerida, este archivo solo contiene una pantalla la cual indica el modo o estado del sistema ya sea conectado o desconectado.

El segundo archivo del sistema es la base de datos en Access, el cual cuenta con una pantalla de ingreso validada por usuario y contraseña, menú principal, además de pantallas de consultas y reportes. Esta es la parte del sistema con la cual el usuario final mantiene contacto. En la Fig. 5 se muestran las relaciones del diseño del sistema de información y la Fig. 6 el diseño de la base de datos.

\section{SLC-500}

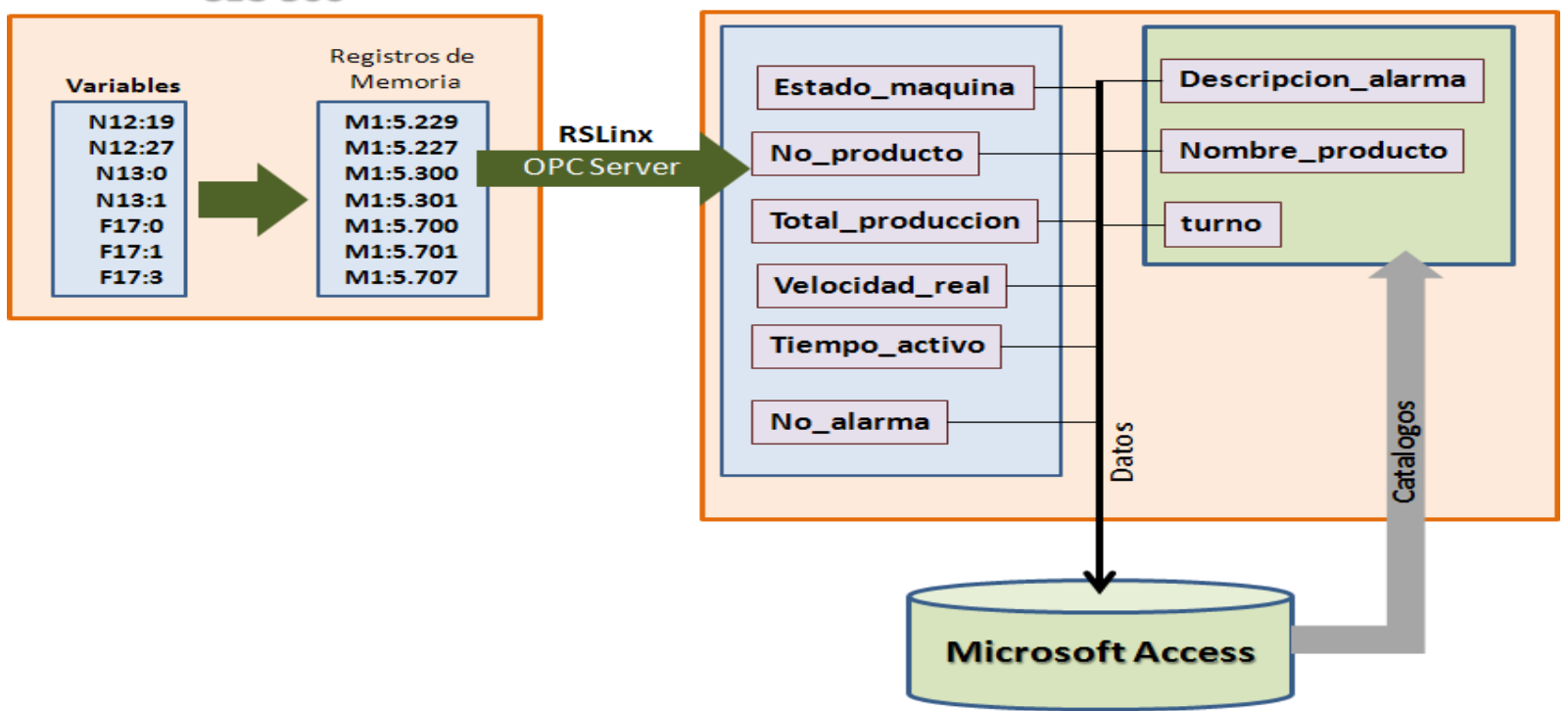

Figura 5. Módulos del sistema de información, fuente: elaboración propia 


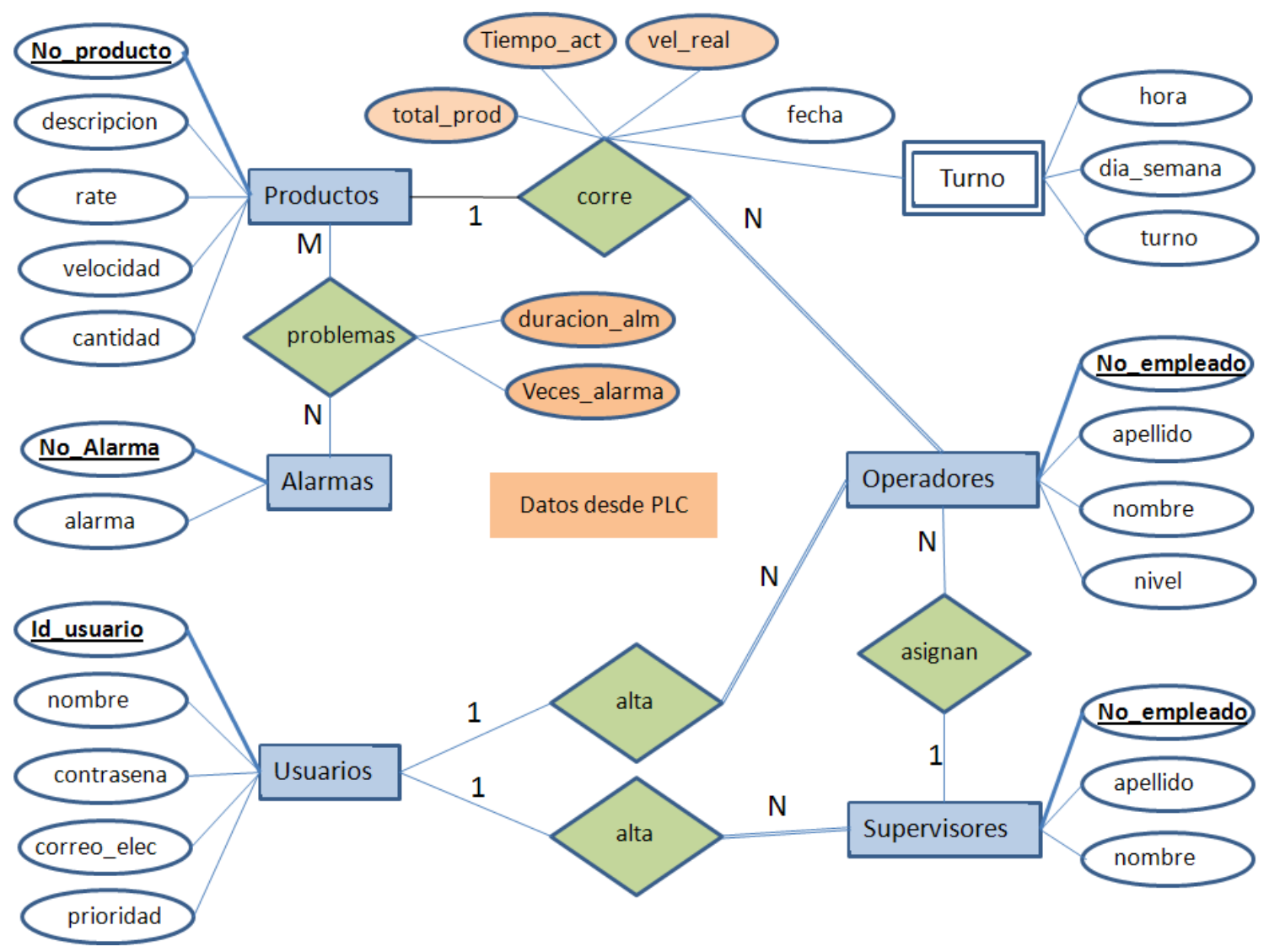

Figura 6. Diagrama entidad relación del sistema de información

Para que el sistema de información pueda leer las variables deseadas del PLC, primero se debe configurar en el programa del PLC la transferencia de valores de variables hacia los registros de memoria de la tarjeta MVI46DNFT, ya que estos registros de memoria son los que serán leídos por el servidor OPC.

Para efectuar esta tarea, primero se tienen que insertar las tres rutinas proporcionadas por Prosoft en el programa de la máquina, elaborado en RSLogix 500, estas rutinas son:

- MVI46 MAIN.

- MVI46 DATA.

- MVI47 CTRL.

El módulo de comunicación debe ser configurado para poder lograr la comunicación del tipo Ethernet, algunas variables que pueden ser configurados son la velocidad de configuración, dirección ip del dispositivo, etc., esto se hace mediante la modificación de dos archivos descargables 
mediante un cable RS232, los archivos son modificados mediante un editor de texto como notepad, para posteriormente ser descargados al módulo.

Comunicación Ethernet de la computadora/servidor.

Uno de los pasos más sencillos, pero que muchas ocasiones son pasado por alto, es la verificación de las propiedades de red de la computadora o servidor, el cual estará en comunicación con el PLC, pues si la configuración no es la adecuada, la comunicación no se establecerá.

Configuración del servidor OPC en RSLinx.

El software RSLinx es el cual estaremos utilizando como servidor OPC, para poder efectuar esta asignación, se debe crear un tópico de comunicación, el cual debe estar direccionado am módulo MVI46-DNFT mediante su dirección ip. Una vez creado el tópico de comunicación, la comunicación puede ser establecida mediante el uso o llamado del nombre del tópico. En esta aplicación el nombre usado para el tópico de comunicación es "link".

Pruebas de comunicación.

Una vez establecida la configuración de la comunicación, se pueden realizar pruebas en la comunicación, esto se puede realizar usando una hoja de Excel, tecleando en las celdas una formula en la forma:

$$
=\text { RSLinx } \mid \text { Topico!'Variable del PLC' }
$$

Base de Datos.

Como se ha mencionado la base de datos fue elaborada en Microsoft Access 2016, esta base de datos debe ser grabada en la dirección, sin sufrir modificaciones en el directorio o nombre del archivo, para eso se debe generar una nueva carpeta:

Módulo de adquisición de datos.

El módulo de adquisición de datos "FF_DAQ_1" elaborado en .NET es un módulo que puede ser ejecutado en forma separada de la base de datos, para esto se debe instalar la carpeta "FF_DAQ_1" en la computadora o servidor la cual fue configurada para la comunicación hacia el PLC, como se ha mencionado en los pasos anteriores de este capítulo.

Procesos del sistema de información. 


\section{Resultados obtenidos}

Beneficios de la propuesta, escenario aplicando el sistema de información

Los PLC son máquinas secuenciales que ejecutan las instrucciones indicadas en el sistema de información almacenado en la memoria, generando órdenes y señales de mando a partir de las señales de entrada leídas de la aplicación, al detectarse cambios en las señales, el PLC reacciones según el programa hasta obtener las órdenes de salida, esta secuencia se ejecuta continuamente para conseguir el control actualizado del proceso; con los sistemas de recolección de datos SCADA a cargo del PLC, el cual tomas las señales y las envía a las estaciones remotas usando un protocolo determinado, entre los beneficios del diseño e implementación del sistema de información se encuentran:

- Disponibilidad de la información en tiempo real y obtención de datos históricos de las variables de producción de la máquina.

- Las tareas de supervisión y control del proceso se han mejorado, el operador puede visualizar en pantalla del computador cada una de las estaciones remotas que conforman el sistema, los estados, las situaciones de alarma y tomar acciones físicas sobre el equipo.

- Obtención de datos relacionados a los tiempos muertos como lo son duración y descripción del evento.

- Envío de alertas a usuarios específicos relacionados a tiempos muertos excesivos para una reacción adecuada los eventos de fallas.

- Disponibilidad del sistema las 24 horas del día.

- Generación y manejo de distintos tipos de reportes.

- Registros de turnos de trabajo, áreas, maquinas, secciones, supervisores, operadores.

- Listas de códigos de productos.

- Listas y descripciones de fallas.

- Generación de niveles diferentes de usuarios del sistema, proporcionando seguridad en la información solo permitiendo utilizar las opciones y pantallas autorizadas para el usuario.

El sistema ha sido desarrollado para cubrir las necesidades detectadas en la empresa, el diseño proporciona la flexibilidad de recolectar datos de la maquina dobladora de sabanas, desarrollar promedios y consultas de los datos sin comprometer la conexión hacia el PLC; visualizando los datos de producción y tiempos muertos relacionando algunas variables como operadores, turnos y productos; buscando tendencias que puedan ayudar a la empresa en la búsqueda de la mejora continua. El sistema ha sido instalado en la empresa, probando y usando todas las opciones desarrolladas; los resultados obtenidos han sido los esperados, proporcionando al personal de 
supervisión los datos requeridos para analizar la problemática de la empresa, y las herramientas necesarias para reaccionar ante estos de mejor manera.

\section{Escenario sin aplicar el sistema de información}

Antes de desarrollar el sistema de información se presentaban problemas en los datos como fallas del equipo, paros, motivos de los paros que se generaban en la máquina dobladora de sábanas; ya que entre un turno de trabajo y otro se reiniciaba la información, por lo que no se podía integrar y analizar.

El desempeño del equipo no era monitoreado en tiempo real por los operadores de la máquina, lo que generaba paros constantes y pérdida de la producción; los tiempos caídos del equipo como resultado de las fallas eran constantes al no existir un monitoreo en tiempo real.

Los registros de producción o manufactura anteriormente se capturaban en un sistema SAP, estos se capturaban desde reportes escritos por los operadores de la maquina dando lugar a una pérdida de tiempo entre el llenado del reporte escrito a mano y la captura en el SAP, por lo que se incrementaban los tiempos muertos de la máquina cuando ocurrían fallas.

El personal que podía proporcionar apoyo al momento de ocurrir problemas en el equipo, no se enteraba de la situación hasta tiempo o turnos después, aumentando el tiempo muerto por problemas de comunicación, disminuyendo la productividad de la máquina.

Por lo que el desarrollo del sistema de información para la adquisición de datos automáticos de la máquina dobladora de sábanas quirúrgicas era una necesidad de alta prioridad.

\section{Operación del sistema de información}

Ya teniendo el sistema instalado, se han definido cuatro grandes pasos para la operación del sistema, que son:

1. Organización y Administración para el funcionamiento del Sistema.

2. Inicialización del Sistema.

3. Conexión del módulo adquisición de datos.

4. Uso de la base de datos, elaboración de consultas y reportes.

La Fig. 7 muestra el inicio del sistema de información, donde se encuentra en línea para la obtención de los datos, del lado derecho de la pantalla se observan datos del turno de trabajo y la velocidad. 


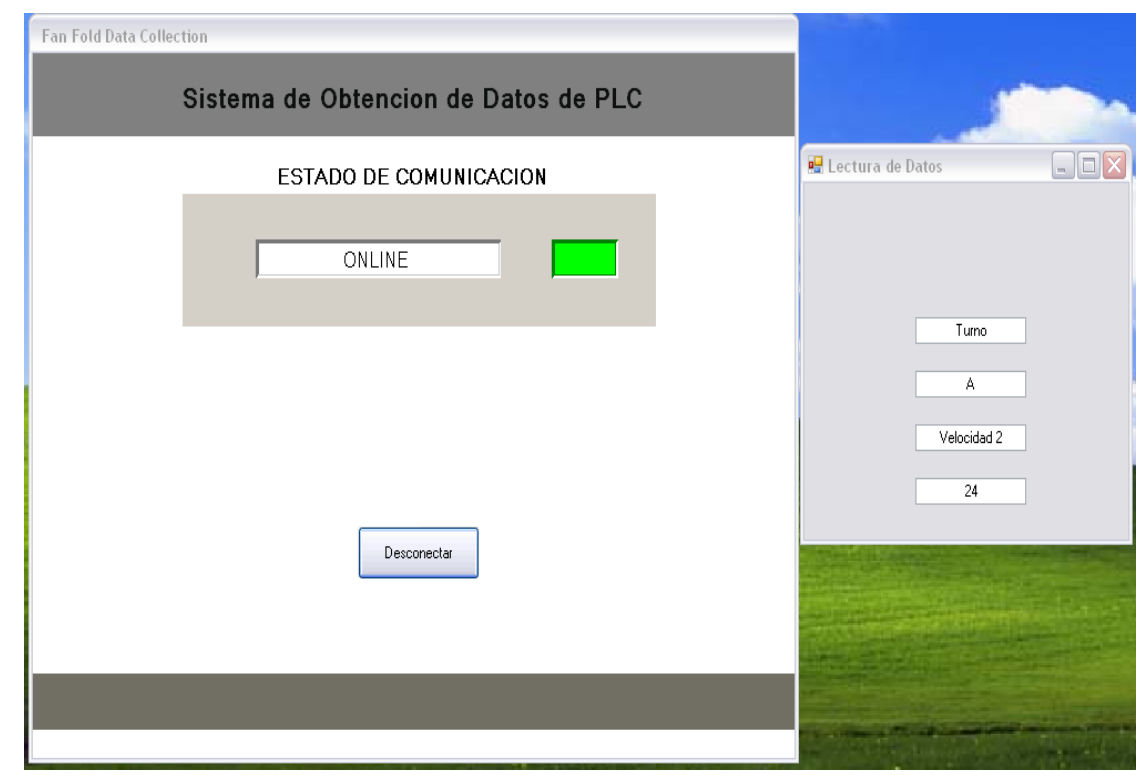

Figura 7. Pantalla inicial del módulo FF_DAQ_1

La Fig. 8 muestra las opciones del menú principal de sistema de información donde se tienen las opciones de Operadores, Supervisores, Códigos, Producción y Alarmas. Los criterios de las relaciones entre estos componentes son:

Además, se consideran los criterios que rigen las relaciones entre estos componentes tales como:

- Un operador debe tener un nombre, apellido, nivel y número de empleado.

- Un supervisor se compone de nombre, apellido y número de empleado

- Un supervisor asigna operadores a la máquina.

- Un usuario debe tener ID, contraseña, nombre, correo electrónico y prioridad (nivel de acceso).

- Un usuario da de alta supervisores y usuarios (según el nivel de acceso).

- Un Turno consta de hora, día de la semana (lunes-domingo), nombre del turno.

- Un Producto consta de número o código del producto, descripción, rate o promedio validado de productos que se esperan obtener por hora, velocidad recomendada, y cantidad de producto por paquete.

- Un operador corre un producto en la maquina en un turno en específico.

- Una Alarma consta de código o número de alarma y descripción (alarma). 
- Una Alarma ocurre cuando se tiene un problema al correr un producto determinado.

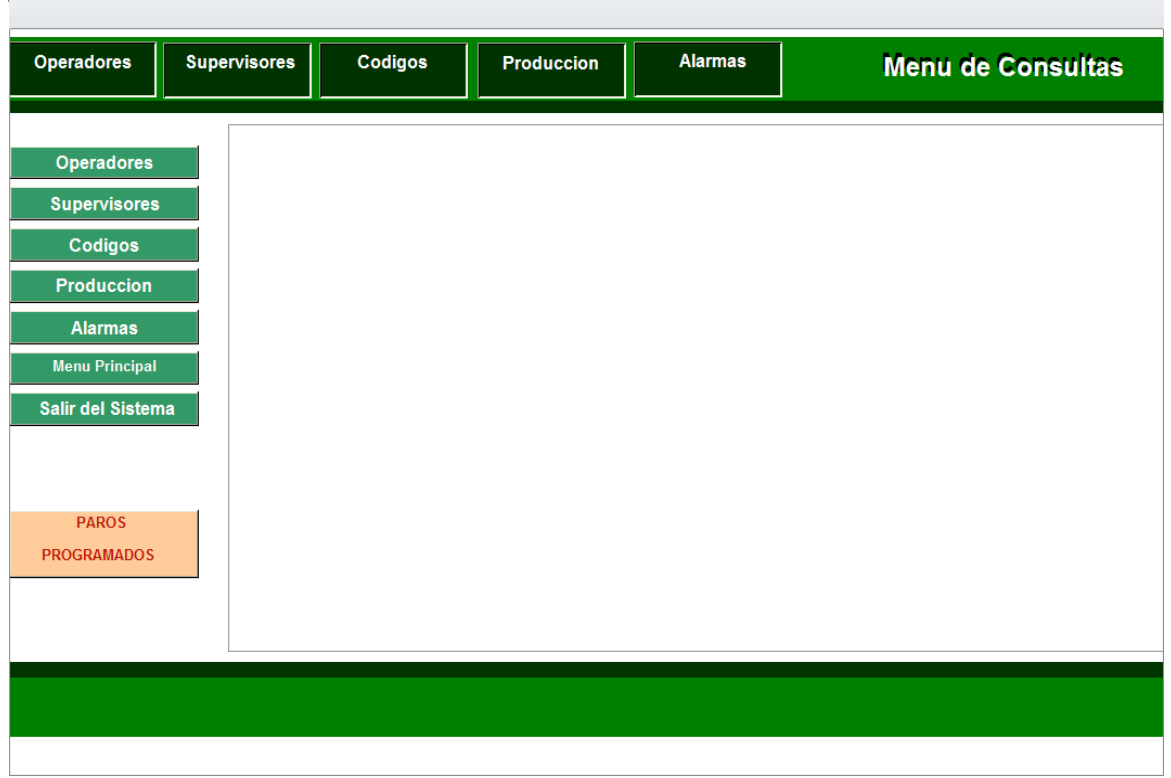

Figura 8. Menú general del sistema de información

La Fig. 9 muestra el reporte de paros programados donde se pueden consultar principalmente cuando se tienen cambios en la planeación de la producción de la máquina dobladora de sábanas.

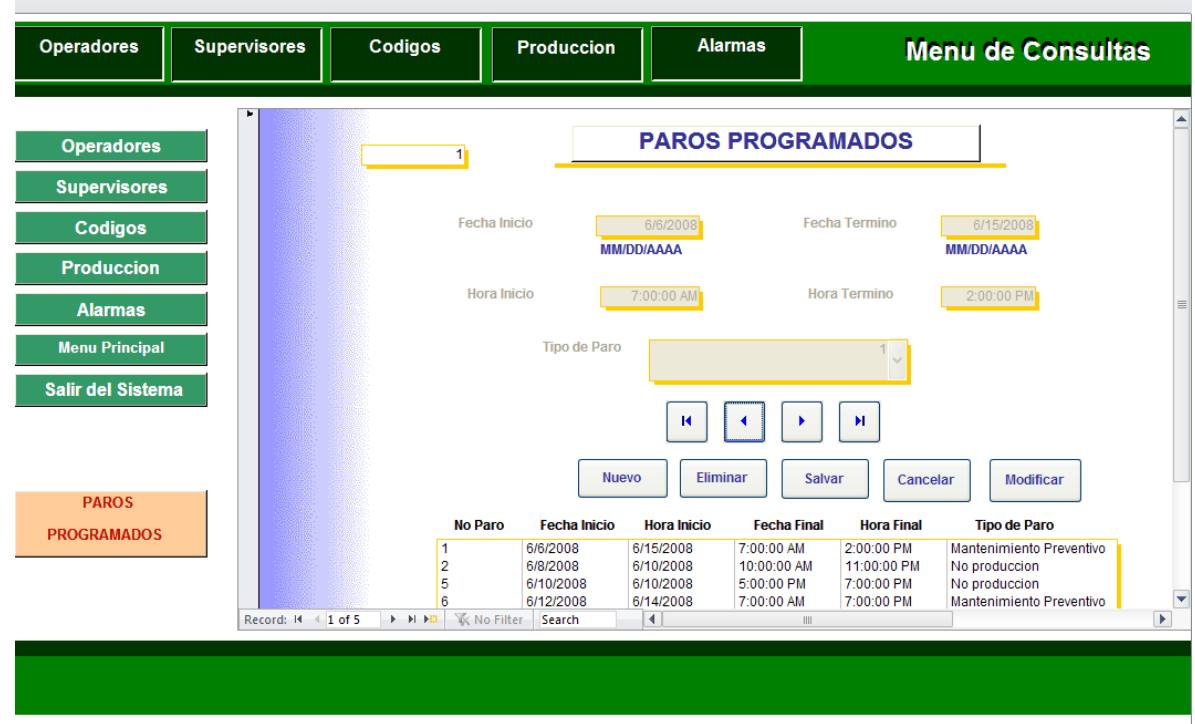

Figura 9. Reporte de paros programados 
En la Fig. 10 se muestra un concentrado de los componentes del sistema de información implementado, entre los que destacan: Los formularios, las tablas de la base de datos, los reportes, módulos y consultas.

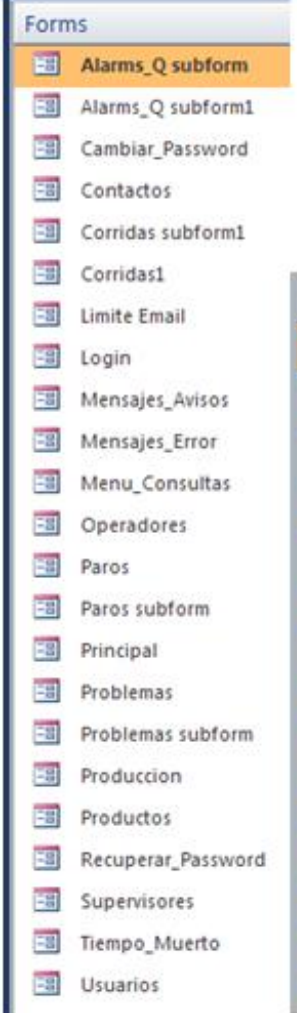

\begin{tabular}{|c|c|}
\hline 中团 & Alarmas \\
\hline 中困 & Contactos \\
\hline 中团 & Corridas \\
\hline 中困 & Limite Email \\
\hline$\Rightarrow$ 柬 & Operadores \\
\hline$\mapsto$ 圅 & Paros \\
\hline 四 & Problemass \\
\hline 中团 & Problems \\
\hline$\Rightarrow$ 目 & Productos \\
\hline 中团 & Supervisores \\
\hline 困 & Table1 \\
\hline 田 & Tipos de Paro \\
\hline 中团 & Turnos \\
\hline 中团 & Usuarios \\
\hline
\end{tabular}

\begin{tabular}{|c|c|}
\hline \multicolumn{2}{|c|}{ Reports } \\
\hline 19 & Alarmas \\
\hline 圆 & Alarms_Q subreport \\
\hline 回 & Contactos \\
\hline 15 & Corridas \\
\hline 19 & Corridas_1 \\
\hline 回 & Corridas_2 \\
\hline 回 & Corridas_3 \\
\hline 19 & Operadores \\
\hline 19 & Paros \\
\hline 圆 & Problemas \\
\hline 19 & Productos \\
\hline 19 & Supervisores \\
\hline 圆 & Usuarios \\
\hline
\end{tabular}

\section{Modules}

is AutoExec

\%8. Form Avisos

15.5 Module1

\section{Queries}

7] Alarms_Q

I] Consultas_paros

I] Query_Usuarios

अ SELECT DISTNNCTRO

Figura 12. Vista de los componentes del sistema de información

\section{Ejemplo del Código en VB.Net}

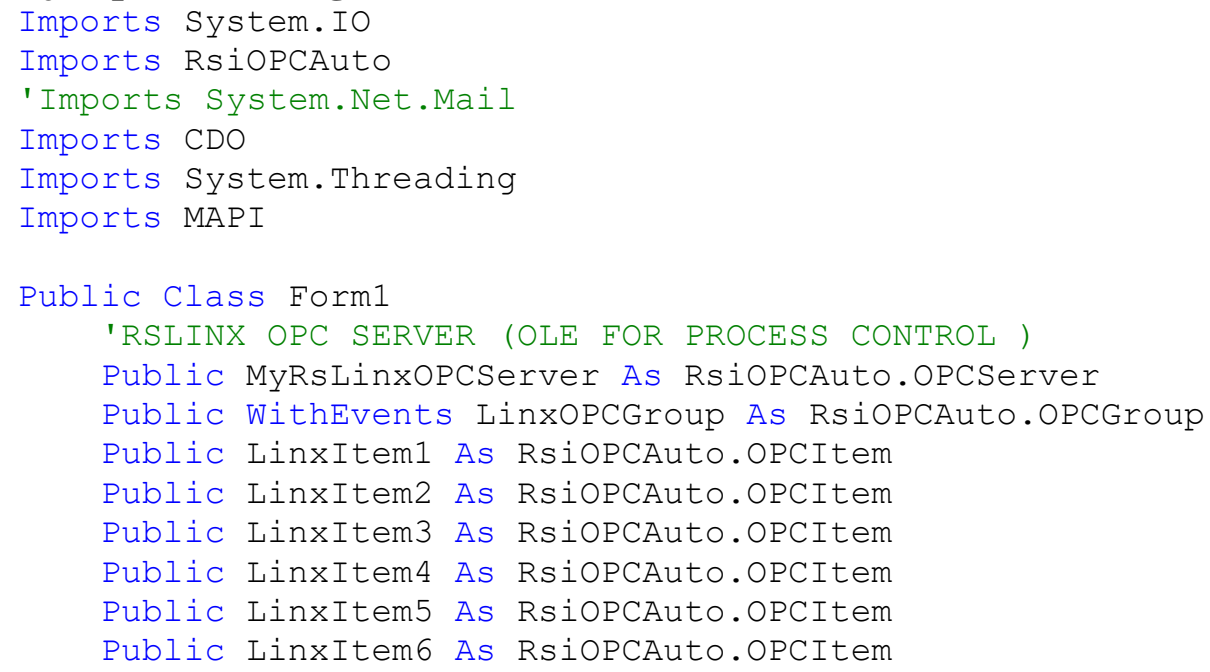




\section{' OLEDB}

Shared OleDbConnection As

System.Data.OleDb.OleDbConnection

Shared TableAdapter As System.Data.OleDb.OleDbDataAdapter

Public Sub Email()

' Variables E-mail prioridad 5

Dim Prioritario, correo5, tiempo5 As object

Dim Str1 As String

Dim Str2 As String

'Variables de Ole

OleDbConnection $=$ New

System.Data.OleDb.OleDbConnection()

OleDbConnection. ConnectionString =

"Provider=Microsoft. Jet. OLEDB.4.0; Data

Source=C: \titulacion \Sistema_Fan_Fold.mdb"

Try

OleDbConnection. Open ()

Catch ex As Exception

MsgBox (ex.Message)

End Try

Str1 = "SELECT * from LimiteEmail WHERE prioridad=5"

Str2 = "SELECT * from contactos order by id_usuario"

Dim OleDbreadcommand As New

System.Data.OleDb.OleDbCommand

OleDbreadcommand.CommandText $=$ Str 1

OleDbreadcommand. Connection = OleDbConnection

Dim OleDbread As System.Data.OleDb.OleDbDataReader

OleDbread = OleDbreadcommand. ExecuteReader ()

Do While OleDbread.Read()

tiempo5 = OleDbread.Item("duracion")

Loop

OleDbConnection.Close ()

OleDbConnection. Open ()

OleDbreadcommand. CommandText $=$ Str2

OleDbreadcommand. Connection = OleDbConnection

'Dim OleDbread As System.Data.OleDb.OleDbDataReader

OleDbread = OleDbreadcommand. ExecuteReader ()

Do While OleDbread.Read()

Prioritario = OleDbread.Item("prioridad")

correo5 = OleDbread.Item("correo_electronico")

If correo5 <> "0" Then

Email1 (correo5)

End If

Loop

End Sub 


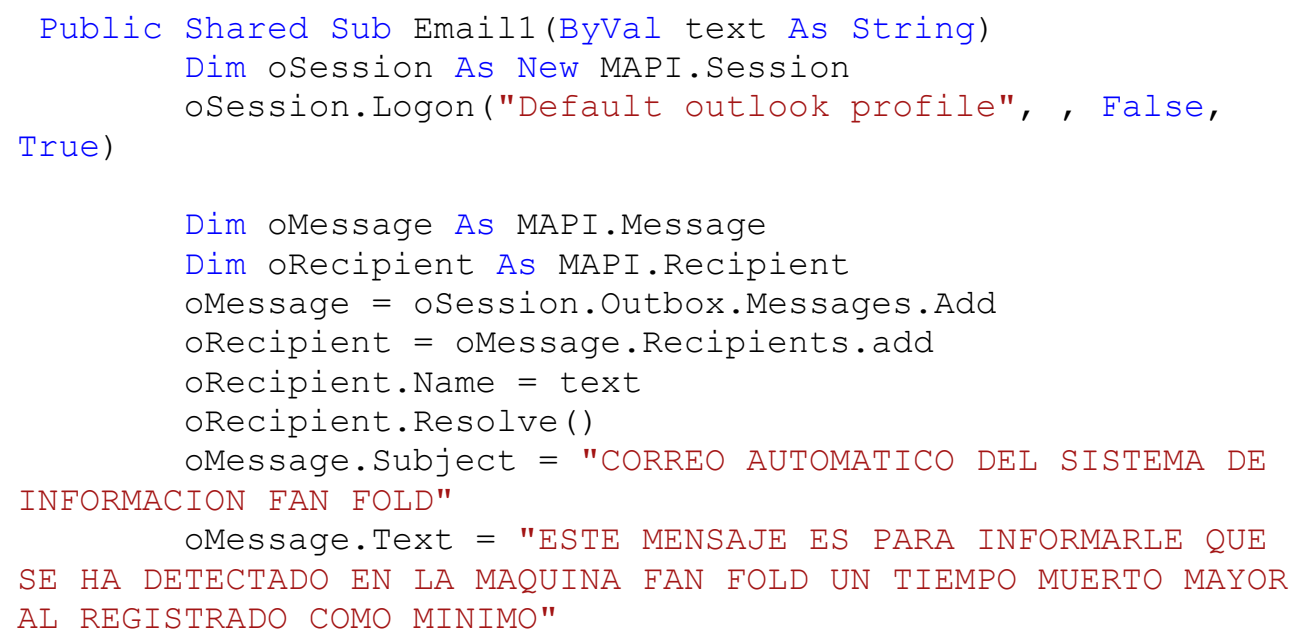

\section{Conclusiones}

Los sistemas de información son un factor clave en las organizaciones y empresas, actualmente se requieren herramientas de software y hardware que puedan ayudar a obtener ventajas competitivas para apoyar en diferentes procesos industriales y de esta manera incidan en la toma de decisiones y en la aplicación de la mejora continua.

La adquisición automática de datos representa un medio importante porque presenta los valores generados y datos históricos del comportamiento de los equipos de fabricación en las empresas de manufactura.

Con el análisis de estos datos se pueden obtener puntos óptimos de producción, ventanas problemáticas en el proceso de fabricación, grado de afectación de la interacción humana, predecir o prevenir problemas operativos, entre otros.

En este proyecto de desarrollo de software de un sistema de información utilizando datos adquiridos de un PLC de una máquina dobladora de sábanas se ha compuesto de varios módulos complejos entre los que destacan: Instalación del módulo ProSoft MVI46-DNFT para la adquisición de datos del PLC, inserción y modificación del programa del PLC, modificación de la lógica de escalera para agregar las variables para su lectura, configuración de la comunicación del módulo ProSoft, comunicación ethernet 
de la computadora al servidor y viceversa, configuración del servidor OPC en el módulo RSLinx, pruebas de comunicación, diseño y creación de la base de datos, programación del módulo en VB.Net para la adquisición de datos, análisis de los datos obtenidos en el software Cognos.

Este sistema de información ha representado un ahorro en recursos de tiempo, personal operativo, procesos industriales y ha optimizado los procesos de fabricación de la maquinaria; al implementar el sistema de adquisición de datos del PLC, han sido obtenidos de manera automática para su proceso en el sistema de información y en los subsecuentes procesos del análisis de la información obtenida para mejorar y prevenir las fallas en el equipo.

Como parte de los resultados obtenidos y de la demostración de las posibilidades y aplicaciones que son posibles de desarrollar, la empresa ha tomado la decisión de llevar el desarrollo del sistema de recolección de datos al siguiente nivel, con el desarrollo aplicado a todo el sector del cuidado de la salud que incluye plantas en los Estados Unidos, México y Honduras, integrando el sistema en un nuevo desarrollo llamado Midas (Management Information and Data Adquisition System) el cual está siendo integrado a la intranet de la empresa.

En cualquier tipo y nivel de empresas se requieren de sistemas de adquisición de datos, estos pueden proporcionarles ventajas competitivas. Si la organización no cuenta con presupuestos para la comprar de sistemas especializados, se pueden desarrollar sistemas como el expuesto que pueden ofrecer los mismos resultados. Además, en nuestro país tenemos la necesidad de preparar personal técnico, para la investigación, diseño, integración y aplicación de sistemas de información con interacción hacia los dispositivos de automatización industrial.

\section{References:}

1. Abdulsattar, N., Mustafa, F., \& Hadi, S. (2019). Design and Implementation of SCADA System for Sugar Production Line. AlKhwarizmi Engineering Journal, 15(2). doi:

https://doi.org/10.22153/kej.2019.01.002

2. Amaya, J. (2018). Sistemas de Información Gerenciales. México: ECOE Ediciones.

3. Bagal, K., Kadu, C., Vikhe, P., \& Parvat, B. (2018). PLC Based Real Time Process Control using SCADA and MATLAB. Paper presented at the 2018 Fourth International Conference on Computing Communication Control and Automation (ICCUBEA), Pune, India.

4. Caicedo, J., Orna, J., Balseca, O., Mayorga, D., Viteri, E., \& Verdugo, C. (2017). Resistencia de Materiales-Ingeniería Mecánica Estudio de Caso:Degradación del Acero ASTM A36 Sometido al Fenómeno de 
Termofluencia. European Scientific Journal, 13(6), 173-189. doi: http://dx.doi.org/10.19044/esj.2017.v13n6p173

5. Carrera, G., Cabrera, J., Medina, C., Tapia, I., Guamán, D., \& Paredes, V. (2019). Evaluación de Riesgos Laborales y Propuesta de un Plan de Prevención de Riesgos y Emergencias en Centros de Salud Pública (CSP). European Scientific Journal, 15(18), 216-237. doi: http://dx.doi.org/10.19044/esj.2019.v15n18p216

6. Dana, N., \& Yendol-Hoppey, D. (2016). The PLC Book. U.S.A.: SAGE Publications LTD.

7. Hermosillo, S. (2019). Sistema Flexible de Control Basado en Controlador Lógico Programable. Maestría, Tecnológico Nacional de México, Chihuahua, México. Retrieved from http://depi.itchihuahua.edu.mx/repositorio_tesis_mmt/GEN2016_2_ GR012019_HERMOSILLO_RODRIGUEZ_SILVIA_ALEJANDRA .pdf

8. Hoffer, J., George, J., \& Valacich, J. (2011). Modern Systems Analysis and Design (7th Edition ed.): Prentice Hall / Pearson Education.

9. Laudon, K., \& Laudon, J. (2012). Sistemas de Información Gerencial. Décimosegunda edición. Mexico, D.F.: Pearson Educación.

10. Mandado, E., Acevedo, J., Fernández, C., \& Armesto, J. (2009). Autómatas Programables y Sistemas de Automatización (Segunda Edición ed.). España: Marcombo, S.A.

11. McLeod, R., \& Schell, G. (2001). Management Information Systems: Prentice Hall.

12. Orna, J., Balseca, O., Caicedo, J., Mayorga, D., Viteri, E., \& Verdugo, C. (2017). Análisis y Diseño de una Aeronave no Tripulada para uso Agrícola. European Scientific Journal, 13(6), 135-156. doi: http://dx.doi.org/10.19044/esj.2017.v13n6p135

13. Ozz, E. (2017). Administración de los Sistemas de Información (7a. Edición ed.). México: Cengage Learning.

14. Paredes, L., Cepeda, R., Mayorga, D., Viteri, E., Barsallo, S., \& Paredes, V. (2019). Estudio de Factibilidad Económica de Medidas de Control de Riesgos Laborales: Área de Lavandería "Hospital Alfredo Noboa Montenegro"-Guaranda. European Scientific Journal, 15(13), 74-94. doi: http://dx.doi.org/10.19044/esj.2019.v15n13p74

15. Pressman, R. (2010). Ingeniería del Software. Un enfoque práctico (Vol. 7a. Edición). México, D.F.: McGraw-Hill.

16. RockwellAutomation. (2021). Rockwell Automation Retrieved 15/01/2021, 2021, from https://www.rockwellautomation.com/enus/products/hardware/allen-bradley.html

17. Rowley, C. (2021). Automation Retrieved 15/01/2021, 2021, from https://www.rowleycompany.com/drapery-fan-folding-device 
18. Silberschatz, A., Korth, H., \& Sudarshan, S. (2002). Fundamentos de Bases de Datos. Madrid, España: McGraw-Hill Inc.

19. Thomas, M., \& McDonalds, J. (2015). Power System SCADA and Smart Grids. U.S.A.: CRC Press Taylor and Francis Group.

20. Tobar, J., Frías, G., Romero, D., \& Barrientos, M. (2019). Análisis de la Gestión de las Tecnologías de la Información (TI) dentro del Plan Estrat;egico Empresarial del Grupo Intercom. European Scientific Journal, 15(22), 150-164. doi:

http://dx.doi.org/10.19044/esj.2019.v15n22p150

21. Tocci, R., \& Widmer, N. (2003). Sistemas Digitales Principios y Aplicaciones. México: Pearson Educación.

22. Yasaca, S., Hidalgo, I., Rodríguez, M., Ceballos, K., Latorre, N., \& Hernández, S. (2019). Análisis Comparativo de las Bibliotecas Virtuales Científicas de la Salud en los Estudiantes Universitarios. European Scientific Journal, 15(13), 360-373. doi: http://dx.doi.org/10.19044/esj.2019.v15n13p360

23. Zhang, Y., Wang, L., Xiang, Y., \& Ten, C. (2015). Power System Reliability Evaluation With SCADA Cybersecurity Considerations. IEEE Transactions on Smart Grid, 6(4), 1707-1721. doi: 10.1109/TSG.2015.2396994 